\title{
WEAK FORMS OF AMENABILITY FOR BANACH ALGEBRAS
}

\author{
H. SAMEA
}

(Received 8 September 2011)

\begin{abstract}
In this paper, the amenability and approximate amenability of weighted $\ell^{p}$-direct sums of Banach algebras with unit, where $1 \leq p<\infty$, are completely characterized. Applications to compact groups and hypergroups are given.
\end{abstract}

2010 Mathematics subject classification: primary 46H20; secondary 43A20.

Keywords and phrases: amenable Banach algebra, approximately amenable Banach algebra, compact group, compact hypergroup.

\section{Introduction}

The notion of approximate amenability of a Banach algebra was introduced by Ghahramani and Loy in [7]. Dales et al. [6] found a necessary and sufficient condition for approximate amenability of Banach algebras, and also proved that the Banach sequence algebras $\ell^{p}(\omega), 1 \leq p<\infty, \omega \in[1,+\infty)^{I}$, are not approximately amenable. The present paper is a continuation of the paper by Dales et al. By a direct method, it is proved that for a family of nonzero Banach algebras $\left\{\mathfrak{A}_{i}\right\}_{i \in I}, \ell^{p}\left(\left(\mathfrak{A}_{i}\right), \omega\right)$ is amenable (respectively, approximately amenable) if and only if $I$ is finite, and for each $i \in I$, $\mathfrak{A}_{i}$ is amenable (respectively, approximately amenable). For another proof, see [5]. The organization of the paper is as follows. Section 2 is devoted to preliminaries and notations which are needed throughout the rest of the paper. Section 3 gives a complete characterization of amenability and approximate amenability for weighted $\ell^{p}$-direct sums of Banach algebras with unit, where $1 \leq p<\infty$. In Section 4 it is proved that for the matrix Banach algebra $\tilde{E}_{p}(I)$, the two notions of amenability and approximate amenability are equivalent. Moreover, applications to compact groups and hypergroups are given. As a corollary, it is proved that if $G$ is an infinite compact group, then the convolution Banach algebra $L^{2}(G)$ is not approximately amenable. This is a generalization of Proposition 2.30 of [1] (see also [2]).

(C) 2011 Australian Mathematical Publishing Association Inc. 0004-9727/2011 \$16.00 


\section{Preliminaries}

Let $A$ be a Banach algebra, and let $X$ be a Banach $A$-bimodule. A derivation is a bounded linear map $D: A \rightarrow X$ such that

$$
D(a b)=D(a) \cdot b+a \cdot D(b) \quad(a, b \in A) .
$$

For $x \in X$, set $\operatorname{ad}_{x}: a \mapsto a \cdot x-x \cdot a, A \rightarrow X$. Then $\operatorname{ad}_{x}$ is a derivation; these are the inner derivations. A derivation $D: A \rightarrow X$ is approximately inner if there is a net $\left(x_{\alpha}\right) \subseteq X$ such that

$$
D(a)=\lim _{\alpha} a \cdot x_{\alpha}-x_{\alpha} \cdot a \quad(a \in A) .
$$

A Banach algebra $A$ is amenable (respectively, approximately amenable) if every derivation from $A$ into $X^{*}$ is inner (respectively, approximately inner) for all Banach $A$-bimodules $X$. For more details see [7, 10, 12].

The following result is taken from [6, Theorem 4.2]. For the definition of $\ell^{p}(\omega)$, see [6] or Definition 3.1 of the present paper.

THEOREM 2.1. The Banach sequence algebras $\ell^{p}(\omega), 1 \leq p<\infty, \omega \in[1,+\infty)^{I}$, are not approximately amenable.

Let $A$ be a Banach algebra. The projective tensor product $A \widehat{\otimes} A$ is a Banach $A$-bimodule, under the operations defined by $c \cdot(a \otimes b)=c a \otimes b$ and $(a \otimes b) \cdot c=$ $a \otimes b c$ for $a, b, c \in A$. The corresponding diagonal operator $\pi_{A}: A \widehat{\otimes} A \rightarrow A$ is defined through $\pi_{A}(a \otimes b)=a b(a, b \in A)$. For more details, see [4].

The following result is a characterization of amenable Banach algebras, and is taken from [10]. See also the comment after Corollary 2.2 of [6].

Theorem 2.2. Let $A$ be a Banach algebra. Then $A$ is amenable if and only if there is a constant $C>0$ such that, for each $\epsilon>0$ and each finite subset $S$ of $A$, there exists $F \in A \otimes A$ with $\|F\|_{\pi} \leq C$ such that, for each $a \in S$ :

(i) $\|a \cdot F-F \cdot a\|_{\pi}<\epsilon$;

(ii) $\left\|a-a \pi_{A}(F)\right\|<\epsilon$.

The following characterization of approximate amenability is taken from [6, Proposition 2.1].

Theorem 2.3. Let $A$ be a Banach algebra. Then $A$ is approximately amenable if and only if, for each $\epsilon>0$ and each finite subset $S$ of $A$, there exist $F \in A \otimes A$ and $u, v \in A$ such that $\pi_{A}(F)=u+v$, and for each $a \in S$ :

(i) $\|a \cdot F-F \cdot a+u \otimes a-a \otimes v\|_{\pi}<\epsilon$;

(ii) $\|a-a u\|<\epsilon$ and $\|a-v a\|<\epsilon$.

\section{Amenability and approximate amenability of $\ell^{p}\left(\left(\mathfrak{H}_{i}\right), \omega\right)(1 \leq p<\infty)$}

Our starting point in this section is the following definition. 
Definition 3.1. Given a set $I$, a family $\left\{\mathfrak{A}_{i}\right\}_{i \in I}$ of Banach algebras, and $\omega=\left(\omega_{i}\right) \in$ $[1,+\infty)^{I}$, define, for $1 \leq p<\infty$,

$$
\ell^{p}\left(\left(\mathfrak{H}_{i}\right), \omega\right)=\left\{\left(\mathfrak{a}_{i}\right): \mathfrak{a}_{i} \in \mathfrak{A}_{i}, \sum_{i \in I} \omega_{i}\left\|\mathfrak{a}_{i}\right\|_{\mathfrak{H}_{i}}^{p}<\infty\right\} .
$$

It is easy to check that $\ell^{p}\left(\left(\mathfrak{A}_{i}\right), \omega\right)$ is a Banach algebra with pointwise multiplication and the norm

$$
\left\|\left(\mathfrak{a}_{i}\right)\right\|_{p, \omega}=\left(\sum_{i \in I} \omega_{i}\left\|\mathfrak{a}_{i}\right\|_{\mathfrak{A}_{i}}^{p}\right)^{1 / p} \quad\left(\left(\mathfrak{a}_{i}\right) \in \ell^{p}\left(\left(\mathfrak{A}_{i}\right), \omega\right)\right) .
$$

The Banach algebra $\ell^{p}\left(\left(\mathfrak{A}_{i}\right), \omega\right)$ is called the weighted $l^{p}$-direct sum of the family $\left(\mathfrak{H}_{i}\right)$ with weight $\omega$. If for each $i \in I, \mathfrak{A}_{i}=\mathfrak{A}$, denote $\ell^{p}\left(\left(\mathfrak{A}_{i}\right), \omega\right)$ by $\ell^{p}(I, \mathfrak{A}, \omega)$. If for each $i \in I, \omega_{i}=1$, denote $\ell^{p}(I, \mathfrak{A}, \omega)$ by $\ell^{p}(I, \mathfrak{A})$. Also define $\ell^{p}(I, \omega)=\ell^{p}(I, \mathbb{C}, \omega)$, $\ell^{p}(I)=\ell^{p}(I, \mathbb{C})$, and $\ell^{p}(\omega)=\ell^{p}(\mathbb{N}, \omega)$.

Lemma 3.2. Given a set $I, 1 \leq p<\infty$, and $\omega \in[1,+\infty)^{I}$, the following assertions are equivalent:

(i) $\ell^{p}(I, \omega)$ is approximately amenable;

(ii) $\ell^{p}(I, \omega)$ is amenable;

(iii) $I$ is finite.

Proof. Let $I$ be infinite. Then there exists an infinite countable subset $I_{0}=\left\{i_{n}\right\}_{n \in \mathbb{N}}$ of $I$. The mapping

$$
\ell^{p}(I, \omega) \rightarrow \ell^{p}(\omega) ; \quad\left(\lambda_{i}\right) \mapsto\left(\lambda_{i_{n}}\right)_{n},
$$

is a continuous epimorphism. But, by Theorem $2.1, \ell^{p}(\mathbb{N}, \omega)$ is not approximately amenable. Therefore, by [7, Proposition 2.2], $\ell^{p}(I, \omega)$ is not approximately amenable.

Obviously, if $I$ is finite, then $\ell^{p}(I, \omega)$ is amenable.

Lemma 3.3. Given a set I, a family $\left\{\mathfrak{U}_{i}\right\}_{i \in I}$ of Banach algebras with unit, and $\omega=\left(\omega_{i}\right) \in$ $[1,+\infty)^{I}$, let $\varpi(i)=\omega_{i}\left\|e_{\mathfrak{H}_{i}}\right\|_{\mathfrak{H}_{i}}^{p}(i \in I)$. Then for $1 \leq p<\infty, \ell^{p}(I, \varpi)$ is a Banach algebra, the mapping

$$
\iota: \ell^{1}(I, \varpi) \rightarrow \ell^{p}\left(\left(\mathfrak{A}_{i}\right), \omega\right) ; \iota(a)=\left(a_{i} e_{\mathfrak{H}_{i}}\right) \quad\left(a=\left(a_{i}\right) \in \ell^{p}(I, \varpi)\right),
$$

is well defined, and there exists a linear map $\Theta$ from $\ell^{p}\left(\left(\mathfrak{A}_{i}\right), \omega\right)$ into $\ell^{p}(I, \varpi)$ such that:

(i) $\|\Theta\|=1$;

(ii) $\Theta(\iota(a))=a\left(a \in \ell^{p}(I, \varpi)\right)$;

(iii) $a \Theta(A)=\Theta(\iota(a) A), \Theta(A) a=\Theta(A \iota(a)) \quad\left(a \in \ell^{p}(I, \varpi), A \in \ell^{p}\left(\left(\mathfrak{H}_{i}\right), \omega\right)\right)$;

(iv) for $a \in \ell^{p}(I, \varpi)$ and $\mathcal{F} \in \ell^{p}\left(\left(\mathfrak{H}_{i}\right), \omega\right) \widehat{\otimes} \ell^{p}\left(\left(\mathfrak{H}_{i}\right), \omega\right)$,

$$
a \cdot(\Theta \otimes \Theta)(\mathcal{F})=(\Theta \otimes \Theta)(\iota(a) \cdot \mathcal{F}),(\Theta \otimes \Theta)(\mathcal{F}) \cdot a=(\Theta \otimes \Theta)(\mathcal{F} \cdot \iota(a)) .
$$


Proof. Since for each $i \in I,\left\|e_{\mathfrak{H}_{i}}\right\|_{\mathfrak{H}_{i}} \geq 1$, we have $\varpi \in[1,+\infty)^{I}$. Thus, $\ell^{p}(I, \varpi)$ is a Banach algebra. It is easy to see that $\iota$ is well defined. Let $i \in I$. By the Hahn-Banach theorem, there exists $\theta_{i} \in \mathfrak{A}_{i}{ }^{*}$ with $\left\|\theta_{i}\right\|=1$ and $\theta_{i}\left(e_{\mathfrak{A}_{i}}\right)=\left\|e_{\mathfrak{A I}_{i}}\right\|_{\mathfrak{A}_{i}}$. Define

$$
\Theta: \ell^{p}\left(\left(\mathfrak{A}_{i}\right), \omega\right) \rightarrow \ell^{p}(I, \varpi) ; \Theta(A)=\left(\frac{1}{\left\|e_{\mathfrak{H}_{i}}\right\|_{\mathfrak{H}_{i}}} \theta_{i}\left(\mathfrak{a}_{i}\right)\right) \quad\left(A=\left(\mathfrak{a}_{i}\right) \in \ell^{p}\left(\left(\mathfrak{A}_{i}\right), \omega\right)\right)
$$

Since $\left\|\theta_{i}\right\|=1 \quad(i \in I), \Theta$ is well defined. The equations in (i) and (ii) are direct consequences of $\left\|\theta_{i}\right\|=1$ and $\theta_{i}\left(e_{\mathfrak{H}_{i}}\right)=\left\|e_{\mathfrak{H}_{i}}\right\|_{\mathfrak{H}_{i}}(i \in I)$. The equations in (iii) and (iv) are proved by an easy calculation. For example, if $a=\left(a_{i}\right) \in \ell^{p}(I, \varpi)$ and $A=\left(\mathfrak{a}_{i}\right) \in$ $\ell^{p}\left(\left(\mathfrak{A}_{i}\right), \omega\right)$, then

$$
\begin{aligned}
a \Theta(A) & =\left(a_{i}\right)\left(\frac{1}{\left\|e_{\mathfrak{H}_{i}}\right\|_{\mathfrak{Q}_{i}}} \theta_{i}\left(\mathfrak{a}_{i}\right)\right)=\left(\frac{a_{i}}{\left\|e_{\mathfrak{H}_{i}}\right\|_{\mathfrak{F}_{i}}} \theta_{i}\left(\mathfrak{a}_{i}\right)\right) \\
& =\left(\frac{1}{\left\|e_{\mathfrak{H}_{i}}\right\|_{\mathfrak{H}_{i}}} \theta_{i}\left(a_{i} \mathfrak{a}_{i}\right)\right)=\Theta\left(\left(a_{i} \mathfrak{a}_{i}\right)\right) \\
& =\Theta\left(\left(a_{i} e_{\mathfrak{I}_{i}}\right)\left(\mathfrak{a}_{i}\right)\right)=\Theta(\iota(a) A) .
\end{aligned}
$$

It follows that, for each $B, C \in \ell^{p}\left(\left(\mathfrak{A}_{i}\right), \omega\right)$,

$$
\begin{aligned}
a \cdot(\Theta \otimes \Theta)(B \otimes C) & =(a \Theta(B)) \otimes \Theta(C)=\Theta(\iota(a) B) \otimes \Theta(C) \\
& =(\Theta \otimes \Theta)(\iota(a) B \otimes C)=(\Theta \otimes \Theta)(\iota(a) \cdot(B \otimes C)),
\end{aligned}
$$

and so for each $\mathcal{F} \in \ell^{p}\left(\left(\mathfrak{H}_{i}\right), \omega\right) \widehat{\otimes} \ell^{p}\left(\left(\mathfrak{H}_{i}\right), \omega\right), a \cdot(\Theta \otimes \Theta)(\mathcal{F})=(\Theta \otimes \Theta)(\iota(a) \cdot \mathcal{F})$.

Given a set $I$ and a family $\left\{\mathfrak{A}_{i}\right\}_{i \in I}$ of Banach algebras with unit, for the subset $I_{0}$ of $I$ let

$$
c_{00}^{I_{0}}\left(\left(\mathfrak{H}_{i}\right)\right)=\left\{\left(\mathfrak{a}_{i}\right): \mathfrak{a}_{i} \in \mathfrak{A}_{i}, \mathfrak{a}_{i}=0 \text { for } i \notin I_{0}\right\},
$$

and define $E_{I_{0}} \in c_{00}^{I_{0}}\left(\left(\mathfrak{A}_{i}\right)\right)$ through $\left(E_{I_{0}}\right)_{i}=e_{\mathfrak{U}_{i}}\left(i \in I_{0}\right)$. These notations are used in the following lemma.

Lemma 3.4. Given a set I, $1 \leq p<\infty$, a family $\left\{\mathfrak{H}_{i}\right\}_{i \in I}$ of Banach algebras with unit, and $\omega \in[1,+\infty)^{I}$, let $\ell^{p}\left(\left(\mathfrak{H}_{i}\right), \omega\right)$ be approximately amenable, $\epsilon>0$, and $S$ be a finite subset of $\ell^{p}\left(\left(\mathfrak{H}_{i}\right), \omega\right)$. Then there exist a finite subset $I_{\epsilon}$ of $I$, and $B^{1}, \ldots, B^{m}, C^{1}, \ldots, C^{m}, U, V \in c_{00}^{I_{\epsilon}}\left(\left(\mathfrak{H}_{i}\right)\right)$ such that, if $\mathcal{F}=\sum_{n=1}^{m} B^{n} \otimes C^{n}$, then $\pi_{\ell^{p}\left(\left(\mathfrak{U}_{i}\right), \omega\right)}(\mathcal{F})=U+V$, and moreover, for each $A \in S$ :

(i) $\|A \cdot \mathcal{F}-\mathcal{F} \cdot A+U \otimes A-A \otimes V\|_{\pi}<\epsilon$;

(ii) $\|A-A U\|_{p, \omega}<\epsilon$ and $\|A-V A\|_{p, \omega}<\epsilon$.

Proof. By Theorem 2.3, there exists $\overline{\mathcal{F}}=\sum_{n=1}^{m} \bar{B}_{n} \otimes \bar{C}_{n} \in \ell^{p}\left(\left(\mathfrak{A}_{i}\right), \omega\right) \otimes \ell^{p}\left(\left(\mathfrak{H}_{i}\right), \omega\right)$, such that $\pi_{\ell^{p}\left(\left(\mathfrak{H}_{i}\right), \omega\right)}(\overline{\mathcal{F}})=\bar{U}+\bar{V}$, and for each $A \in S$ :

(i') $\quad\|A \cdot \overline{\mathcal{F}}-\overline{\mathcal{F}} \cdot A+\bar{U} \otimes A-A \otimes \bar{V}\|_{\pi}<\epsilon / 2 ;$

(ii') $\|A-A \bar{U}\|_{p, \omega}<\epsilon / 2$ and $\|A-\bar{V} A\|_{p, \omega}<\epsilon / 2$. 
Let $\epsilon_{1}=\epsilon /\left(8 \max _{A \in S}\left(\|A\|_{p, \omega}+1\right)\right)$. By continuity of the tensor product and the definition of $\|\cdot\|_{p, \omega}$, there exists a finite subset $I_{\epsilon}$ of $I$ such that

$$
\left\|\sum_{n=1}^{m}\left(\bar{B}_{n} E_{I_{\epsilon}}\right) \otimes\left(\bar{C}_{n} E_{I_{\epsilon}}\right)-\sum_{n=1}^{m} \bar{B}_{n} \otimes \bar{C}_{n}\right\|_{\pi}<\epsilon_{1}
$$

and

$$
\left\|\bar{U} E_{I_{\epsilon}}-\bar{U}\right\|_{p, \omega},\left\|\bar{V} E_{I_{\epsilon}}-\bar{V}\right\|_{p, \omega}<\epsilon_{1} .
$$

Let $B_{n}=\bar{B}_{n} E_{I_{\epsilon}}, C_{n}=\bar{C}_{n} E_{I_{\epsilon}}(1 \leq n \leq m), \mathcal{F}=\sum_{n=1}^{m} B_{n} \otimes C_{n}, U=\bar{U} E_{I_{\epsilon}}$, and $V=\bar{V} E_{I_{\epsilon}}$. Then (i') and (ii') give (i) and (ii).

Proposition 3.5. Given a set I, a family $\left\{\mathfrak{A}_{i}\right\}_{i \in I}$ of Banach algebras with unit, and $\omega=\left(a_{i}\right) \in[1,+\infty)^{I}$, if the Banach algebra $\ell^{p}\left(\left(\mathfrak{H}_{i}\right), \omega\right)$ is approximately amenable, then I is finite.

Proof. The notations of Lemmas 3.3 and 3.4 are used. Let $\epsilon>0$ and $S$ be a finite subset of $\ell^{p}(I, \varpi)$. Since $\iota(S)$ is a finite subset of $\ell^{p}\left(\left(\mathfrak{H}_{i}\right), \omega\right)$, there exist by Lemma 3.4 a finite subset $I_{\epsilon}$ of $I$, and $B_{1}, \ldots, B^{m}, C^{1}, \ldots, C^{m}, U, V \in c_{00}^{I_{\epsilon}}\left(\left(\mathfrak{A}_{i}\right)\right)$ such that, if $\mathcal{F}=\sum_{n=1}^{m} B^{n} \otimes C^{n}$, then $\pi_{\ell^{p}\left(\left(\mathfrak{H}_{i}\right), \omega\right)}(\mathcal{F})=U+V$, and for each $a \in S$ :

(i) $\quad\|\iota(a) \cdot \mathcal{F}-\mathcal{F} \cdot \iota(a)+U \otimes \iota(a)-\iota(a) \otimes V\|_{\pi}<\epsilon$;

(ii) $\|\iota(a)-\iota(a) U\|_{p, \omega}<\epsilon$ and $\|\iota(a)-V \iota(a)\|_{p, \omega}<\epsilon$.

For $i \in I$, let $\Theta_{i}$ be the $i$ th component of $\Theta$ (that is, in the notation of the proof of Lemma 3.3, $\left.\Theta_{i}=\left(1 /\left\|e_{\mathfrak{I}_{i}}\right\|_{\mathfrak{A}_{i}}\right) \theta_{i}\right)$. Let

$$
\lambda_{n, i}=\Theta_{i}\left(B^{n}{ }_{i} C^{n}{ }_{i}\right)-\Theta_{i}\left(B^{n}{ }_{i}\right) \Theta_{i}\left(C^{n}{ }_{i}\right) \quad\left(1 \leq n \leq m, i \in I_{\epsilon}\right)
$$

and

$$
F=(\Theta \otimes \Theta)(\mathcal{F})+\sum_{n=1}^{m} \sum_{i \in I_{\epsilon}} \lambda_{n, i} \delta_{i} \otimes \delta_{i},
$$

where $\delta_{i} \in \ell^{p}(I, \varpi)$ is defined by $\delta_{i}(i)=1$ and $\delta_{i}(j)=0 \quad(j \neq i)$. Obviously, $F \in$ $\ell^{p}(I, \varpi) \otimes \ell^{p}(I, \varpi)$. Let $u=\Theta(U)$ and $v=\Theta(V)$. It is clear that

$$
a \cdot\left(\delta_{i} \otimes \delta_{i}\right)=\left(\delta_{i} \otimes \delta_{i}\right) \cdot a \quad\left(a \in \ell^{p}(I, \varpi), i \in I\right),
$$

and so by Lemma 3.3(iv), for each $a \in \ell^{p}(I, \varpi)$,

$$
\begin{aligned}
a \cdot F-F \cdot a & =a \cdot(\Theta \otimes \Theta)(\mathcal{F})-(\Theta \otimes \Theta)(\mathcal{F}) \cdot a \\
& =(\Theta \otimes \Theta)(\iota(a) \cdot \mathcal{F}-\mathcal{F} \cdot \iota(a)) .
\end{aligned}
$$

Thus, by (i) in this proof and Lemma 3.3(ii) and (i), for each $a \in S$, $\|a \cdot F-F \cdot a+u \otimes a-a \otimes v\|_{\pi}=\|(\Theta \otimes \Theta)(\iota(a) \cdot \mathcal{F}-\mathcal{F} \cdot \iota(a)-U \otimes \iota(a)-\iota(a) \otimes V)\|_{\pi}$ $\leq\|\iota(a) \cdot \mathcal{F}-\mathcal{F} \cdot \iota(a)-U \otimes \iota(a)-\iota(a) \otimes V\|_{\pi}<\epsilon$. 
Also, by (ii) and Lemma 3.3(i),

$$
\|a-a u\|_{p, \varpi}=\|\Theta(\iota(a)-\iota(a) U)\|_{p, \varpi} \leq\|\iota(a)-\iota(a) U\|_{p, \omega}<\epsilon,
$$

and similarly $\|a-v a\|_{p, \varpi}<\epsilon$. Moreover,

$$
\begin{aligned}
\pi_{\ell^{p}(I, \varpi)}(F) & =\sum_{n=1}^{m} \Theta\left(B^{n}\right) \Theta\left(C^{n}\right)+\sum_{n=1}^{m} \sum_{i \in I_{\epsilon}} \lambda_{n, i} \delta_{i} \delta_{i} \\
& =\sum_{n=1}^{m} \sum_{i \in I_{\epsilon}} \Theta_{i}\left(B^{n}{ }_{i}\right) \Theta_{i}\left(C^{n}{ }_{i}\right) \delta_{i}+\sum_{n=1}^{m} \sum_{i \in I_{\epsilon}} \lambda_{n, i} \delta_{i} \\
& =\sum_{n=1}^{m} \sum_{i \in I_{\epsilon}} \Theta_{i}\left(B^{n}{ }_{i} C^{n}{ }_{i}\right) \delta_{i}=\sum_{n=1}^{m} \Theta\left(B^{n} C^{n}\right) \\
& =\Theta\left(\pi_{\ell p\left(\left(\mathfrak{I}_{i}\right), \omega\right)}(\mathcal{F})\right)=\Theta(U+V)=u+v .
\end{aligned}
$$

Therefore, by Theorem 2.3, $\ell^{p}(I, \varpi)$ is approximately amenable. Hence, by Lemma 3.2, $I$ is finite.

REMARK 3.6. If, for each $i \in I, \mathfrak{A}_{i}$ has a nonzero character $\phi_{i}$, then there is a simple proof for the above proposition. To see this, suppose that $\ell^{p}\left(\left(\mathfrak{H}_{i}\right), \omega\right)$ is approximately amenable. Define

$$
\Theta: \ell^{p}\left(\left(\mathfrak{A}_{i}\right), \omega\right) \rightarrow \ell^{p}(I, \varpi) ;\left(\mathfrak{a}_{i}\right) \mapsto\left(\phi_{i}\left(\mathfrak{a}_{i}\right)\right),
$$

where $\varpi_{i}=\omega_{i} /\left\|\phi_{i}\right\|^{p}(i \in I)$. Note that for each $i \in I,\left\|\phi_{i}\right\| \leq 1$ (see [4, Section 16]), and so $\varpi_{i} \geq 1$. Clearly $\Theta$ is a bounded linear map. For each $i \in I$, there is $\mathfrak{a}_{i}^{0} \in \mathfrak{A}_{i}$ with $\left\|\mathfrak{a}_{i}^{0}\right\|_{\mathfrak{Q}_{i}}=1$, such that $\left|\phi_{i}\left(\mathfrak{a}_{i}^{0}\right)\right| \geq \frac{1}{2}\left\|\phi_{i}\right\|$. Let $a:=\left(\lambda_{i}\right) \in \ell^{p}(I, \varpi)$. Then it is easy to show that if $A=\left(\left(\lambda_{i} / \phi_{i}\left(\mathfrak{a}_{i}^{0}\right)\right) \mathfrak{a}_{i}^{0}\right)$, then $A \in \ell^{p}\left(\left(\mathfrak{A}_{i}\right), \omega\right)$, and $\Phi(A)=a$. It follows that $\Phi$ is a continuous epimorphism. Hence, by [7, Proposition 2.2], $\ell^{p}(I, \varpi)$ is approximately amenable, and so by Lemma 3.2, $I$ is finite.

Lemma 3.7. Given a set I, a family $\left\{\mathfrak{H}_{i}\right\}_{i \in I}$ of Banach algebras, and $\omega \in[1,+\infty)^{I}$, if $1 \leq p<\infty$, and $\ell^{p}\left(\left(\mathfrak{H}_{i}\right), \omega\right)$ is amenable (respectively, approximately amenable), then, for each $i \in I, \mathfrak{A}_{i}$ is amenable (respectively, approximately amenable).

Proof. For each $i \in I$, the mapping $\pi_{i}: \ell^{p}\left(\left(\mathfrak{H}_{i}\right), \omega\right) \rightarrow \mathfrak{A}_{i} ;\left(\mathfrak{a}_{i}\right) \mapsto \mathfrak{a}_{i}$ is a bounded algebra homomorphism. By [12, Proposition 2.3.1] (respectively, [7, Proposition 2.2]), $\mathfrak{A}_{i}$ is amenable (respectively, approximately amenable).

The following result is the main theorem of the present paper.

TheOREM 3.8. Given a set I, a family $\left\{\mathfrak{H}_{i}\right\}_{i \in I}$ of Banach algebras with unit, and $\omega=\left(a_{i}\right) \in[1,+\infty)^{I}$, if $1 \leq p<\infty$, then the following statements are equivalent.

(i) $\ell^{p}\left(\left(\mathfrak{H}_{i}\right), \omega\right)$ is amenable (respectively, approximately amenable).

(ii) The set I is finite, and, for each $i \in I, \mathfrak{A}_{i}$ is amenable (respectively, approximately amenable). 
Proof. (i) $\Rightarrow$ (ii) is a consequence of Proposition 3.5 and Lemma 3.7.

(ii) $\Rightarrow$ (i) follows from [12, Corollary 2.3.19] (where, for each $i \in I, \mathfrak{A}_{i}$ is amenable), and [7, Proposition 2.7] (where, for each $i \in I, \mathfrak{A}_{i}$ is approximately amenable).

\section{Applications to compact groups and hypergroups}

Let $H$ be an $n$-dimensional Hilbert space and suppose that $B(H)$ is the space of all linear operators on $H$. For $E \in B(H)$, let $\left(\lambda_{1}, \ldots, \lambda_{n}\right)$ be the sequence of eigenvalues of the operator $|E|$, written in any order. Define $\|E\|_{\varphi_{p}}=\left(\sum_{i=1}^{n}\left|\lambda_{i}\right|^{p}\right)^{1 / p} \quad(1 \leq p<\infty)$. For more details, see [8, Definition D.37 and Theorem D.40].

Let $I$ be an arbitrary index set. For each $i \in I$, let $H_{i}$ be a finite-dimensional Hilbert space of dimension $d_{i}$, and let $a_{i} \geq 1$ be a real number. Define

$$
\mathfrak{E}_{p}(I)=\ell^{p}\left(\left(\left(B\left(H_{i}\right),\|\cdot\|_{\varphi_{p}}\right)\right),\left(a_{i}\right)\right) \quad(1 \leq p<\infty) .
$$

This definition is taken from [8, Section 28], using the notation of Definition 3.1.

By [12, Example 2.3.16], for each $i \in I$, the Banach algebra $B\left(H_{i}\right)$ is amenable. Hence Theorem 3.8 yields the following result.

Proposition 4.1. Let $1 \leq p<\infty$. The following statements are equivalent.

(i) $\mathfrak{E}_{p}(I)$ is approximately amenable.

(ii) $\mathfrak{E}_{p}(I)$ is amenable.

(iii) I is finite.

Let $K$ be a compact hypergroup (as defined by Jewett [9]), and $\widehat{K}$ be the set of equivalence classes of continuous irreducible representations of $K$ (see [3], [9, Section 11.3], and [13]). For each $\pi \in \widehat{K}$, let $H_{\pi}$ be the representation space of $\pi$ and $d_{\pi}=\operatorname{dim} H_{\pi}$. By [13, Theorem 2.2], $d_{\pi}<\infty$. Furthermore, by the proof of [13, Theorem 2.2], there exists a constant $c_{\pi}$ such that for each $\xi \in H_{\pi}$ with $\|\xi\|=1$,

$$
\int_{K}|\langle\pi(x) \xi, \xi\rangle|^{2} d \omega_{K}(x)=c_{\pi} .
$$

Let $k_{\pi}=c_{\pi}^{-1}$. By [13, Theorem 2.6], $k_{\pi} \geq d_{\pi}$. Moreover, if $K$ is a group, then $k_{\pi}=d_{\pi}$. The Banach algebras $\mathfrak{E}_{p}(\widehat{K})$, for $p \in[1, \infty)$, are defined with each $a_{\pi}=k_{\pi}$.

Proposition 4.2. Let $K$ be a compact hypergroup, and $1 \leq p<\infty$. The following statements are equivalent.

(i) $\mathfrak{E}_{p}(\widehat{K})$ is approximately amenable.

(ii) $\mathfrak{E}_{p}(\widehat{K})$ is amenable.

(iii) $K$ is finite.

Proof. If $\widehat{K}$ is finite, then $\mathfrak{E}_{2}(\widehat{K})$ is finite-dimensional. So by [13, Theorem 3.4 ], $L^{2}(K)$ is finite-dimensional, and so is $C(K)$. From the comment on [11, p. 57] it follows that $K$ is finite. By Proposition 4.1, the proof is complete. 
If $K$ is a compact hypergroup, then by [3, Theorem 1.3.28], $K$ admits a left Haar measure. Throughout the present paper we use the normalized Haar measure $\omega_{K}$ on the compact hypergroup $K$ (that is, $\omega_{K}(K)=1$ ). Note that by [13, Theorem 3.4], the convolution Banach algebra $L^{2}(K)$ is isometrically algebra isomorphic with $\mathfrak{E}_{2}(\widehat{K})$. Thus the following result is a corollary of the above proposition.

Corollary 4.3. Let $K$ be a compact hypergroup. The following statements are equivalent.

(i) The convolution Banach algebra $L^{2}(K)$ is approximately amenable.

(ii) The convolution Banach algebra $L^{2}(K)$ is amenable.

(iii) $K$ is finite.

As a further corollary, the following generalization of [1, Proposition 2.30] (see also [2]) is obtained.

Corollary 4.4. Let $G$ be an infinite compact group. Then the convolution Banach algebra $L^{2}(G)$ is not approximately amenable.

If $f \in L^{1}(K)$ and $\sum_{\pi \in \widehat{K}} k_{\pi}\|\widehat{f}(\pi)\|_{\varphi_{1}}<\infty$ (where $\widehat{f} \in \mathfrak{E}(\widehat{K})$ is the Fourier transform of $f$, defined by $\widehat{f_{\pi}}=\int_{K} f(x) \pi(\bar{x}) d \omega_{K}(x)(\pi \in \widehat{K})$ ), we say that $f$ has an absolutely convergent Fourier series. The set of all functions with absolutely convergent Fourier series is denoted by $A(K)$ and called the Fourier space of $K$. For $f \in A(K)$ we define $\|f\|_{A(K)}=\| \widehat{f}_{1}$. By [13, Proposition 4.2], $A(K)$ with the convolution product is a Banach algebra and isometrically isomorphic with $\mathfrak{E}_{1}(\widehat{K})$. See also [8] for further results about compact groups. Proposition 4.1 yields the following result.

Corollary 4.5. Let $K$ be a compact hypergroup. The following statements are equivalent.

(i) The convolution Banach algebra $A(K)$ is approximately amenable.

(ii) The convolution Banach algebra $A(K)$ is amenable.

(iii) $K$ is finite.

\section{Acknowledgements}

The author would like to thank the referee for invaluable comments. The author would also like to thank the University of Bu-Ali Sina (Hamedan) for its support.

\section{References}

[1] P. Bharucha, Amenability properties and their consequences in Banach algebras, PhD Thesis, Australian National University, Australia, 2008.

[2] P. Bharucha and R. J. Loy, 'Approximate and weak amenability of certain Banach algebras', Studia. Math. 197(2) (2010), 195-204.

[3] W. R. Bloom and H. Heyer, Harmonic Analysis of Probability Measures on Hypergroups (Walter de Gruyter, Berlin, 1995).

[4] F. Bonsall and J. Duncan, Complete Normed Algebras (Springer, New York, 1973). 
[5] Y. Choi and F. Ghahramani, 'Approximate amenability of Schatten classes, Lipschitz algebras and second duals of Fourier algebras', Q. J. Math. 62(1) (2011), 39-58.

[6] H. G. Dales, R. J. Loy and Y. Zhang, 'Approximate amenability for Banach sequence algebras', Studia Math. 177 (2006), 81-96.

[7] F. Ghahramani and R. J. Loy, 'Generalized notions of amenability', J. Funct. Anal. 208(1) (2004), 229-260.

[8] E. Hewitt and K. A. Ross, Abstract Harmonic Analysis, Vol. II (Springer, Berlin, 1970).

[9] R. I. Jewett, 'Spaces with an abstract convolution of measures', Adv. Math. 18 (1975), 1-110.

[10] B. E. Johnson, 'Cohomology in Banach algebras', Mem. Amer. Math. Soc. 127 (1972).

[11] G. J. Murphy, $C^{*}$-Algebras and Operator Theory (Academic Press, San Diego, CA, 1990).

[12] V. Runde, Lectures on Amenability, Lecture Notes in Mathematics, 1774 (Springer, Berlin, 2002).

[13] R. C. Vrem, 'Harmonic analysis on compact hypergroups', Pacific J. Math. 85(1) (1979), $239-251$.

H. SAMEA, Department of Mathematics, Bu-Ali Sina University, Hamedan, Iran e-mail: h.samea@basu.ac.ir 